\title{
Improving pain management in an hospital ward by implementing an educational intervention: A prospective cohort study
}

\author{
maayan ben sasson ${ }^{1}$, Ofir Hagalili ${ }^{2}$, Elad Schiff ${ }^{2}$, Samuel Attias ${ }^{2}$, and Simon Vulfsons ${ }^{1}$ \\ ${ }^{1}$ Rambam Health Care Campus \\ ${ }^{2}$ Bnei Zion Hospital
}

June 23, 2021

\begin{abstract}
Introduction: the Pain management in internal medicine ward is a common problem. Studies have shown that the evaluation of pain and its documentation by the medical staff is inaccurate and tends to underestimate the level of pain reported by patients. In addition, Physicians often report lack of knowledge and misconceptions regarding pain management. Objectives: To assess the impact of a structured pain educational program on knowledge, skills and abilities [KSA's] of internal medicine ward physicians in pain management. Methods: Eleven physicians from Internal medicine ward B at Bnai-Zion Medical Center, a tertiary academic center in Israel, participated in a pain educational program. Questioners regarding pain KSA's, were completed before and after the educational intervention. Concomitantly, a pain management protocol was implemented in the department. Results: There was a $55.5 \%$ (P.value $=0.003$ ) improvement in the overall satisfaction of the physicians regarding pain management in the internal ward on a 1 to 6 Likert scale (1-strongly disagree, 6-strongly agree) .In a modified KnowPain50 [KP50] questionnaire that assesses physicians KSA, the average score for all physicians improved by $15.3 \%(\mathrm{P} . \mathrm{value}=0.012)$ after the intervention. Conclusion: A targeted educational intervention on pain management can improve physician's KSA, and this we belive may lead to a better treatment for patients suffering from pain in the hospital scenerio.
\end{abstract}

Improving pain management in an hospital ward by implementing an educational intervention: A prospective cohort study

\section{Abstract}

Introduction: Pain management in the internal medicine ward is a common problem. Studies have shown that the evaluation of pain and its documentation by the medical staff is inaccurate and tends to underestimate the level of pain reported by patients. In addition, Physicians often report lack of knowledge and misconceptions regarding pain management.

Objectives : To assess the impact of a structured pain educational program on knowledge, skills and abilities [KSA's] of internal medicine ward physicians in pain management.

Methods : Eleven physicians from Internal medicine ward B at Bnai-Zion Medical Center, a tertiary academic center in Israel, participated in a pain educational program. Questioners regarding pain KSA's, were completed before and after the educational intervention. Concomitantly, a pain management protocol was implemented in the department.

Results : There was a $55.5 \%$ (P.value $=0.003$ ) improvement in the overall satisfaction of the physicians regarding pain management in the internal ward on a 1 to 6 Likert scale (1-strongly disagree, 6-strongly agree) .In a modified KnowPain50 [KP50] questionnaire that assesses physicians KSA, the average score for all physicians improved by $15.3 \%($ P.value $=0.012)$ after the intervention. 
Conclusion : A targeted educational intervention on pain management can improve physician's KSA, and this we belive may lead to a better treatment for patients suffering from pain in the hospital scenerio.

Keywords : Education, Pain Training Programs, pain management

\section{Introduction}

Pain is the most common complaint for which people seek medical care ${ }^{1}$. Pain in the internal medicine ward is common; arising from different diagnoses, and sometimes standing alone as a chronic pain syndrome. Despite its wide variety and high frequency, pain management is often insufficient. Lack of knowledge, suspicion or disbelief to patients complaints and prejudice that pain is not a top priority in treatment ${ }^{2}$ might be the reason for that.

Effective pain management is divided into two important steps: The first step is its assessment and documentation. It has been observed that data collection and reporting by the staff was inaccurate and tended to underestimate the level of pain reported by patients ${ }^{3}$. A difference of nearly two points in a $0-10$ visual analog scale was observed between the intensity of pain reported by patients to the score recorded by the nursing staff ${ }^{3}$. The second step, which is the implementation of the appropriate treatment to the specific type and intensity of pain. Several studies have shown that pain management is insufficient might be due to lack of knowledge and misconceptions regarding pain management and medication, specifically the use of opioids ${ }^{4}$. Although almost $35 \%$ of patients suffer from severe pain during hospitlzition ${ }^{5}$, patients in the internal medicine wards are not properly evaluated and are undertreated for their pain. Studies that assess the knowledge, skills and abilities (KSA) of physicians from different medical fields, showed that pain management is inadequate and is lacking ${ }^{6-7}$. That might lead to unnecessary patient suffering and astronomic high costs for the health system ${ }^{8}$.

It is evident that there is a need for a better physician education in this field. We developed a targeted educational intervention in order to address physician's inappropriate knowledge, skills and attitude regarding pain management. The aim of this study was to evaluate the influence of that process, on internal department physician's KSA.

Methods: This was a prospective cohort study without a randomization or control group. 11 Physician (9 interns who were in different stages of internship and 2 young specialists) from an internal ward department at Bnai-Zion Medical Center, a tertiary academic center in Israel, participated. The Institutional Ethics Committee in accordance with the Declaration of Helsinki approved the study (0138-14-BNZ, NIHNCT02577328)

Research process:

Figure 1 shows the research methodology and the process of the study.

\section{Stage 1- educational intervention}

A thinking task force that structured the educational intervention and its assessment was composed of the Director of the Internal Medicine Department, an intern, a nurse who served as pain trustees in the department, and pain specialists from the Institue for Pain Medicine at Rambam medical center in Haifa, Israel. This cooperation led to the development of the educational intervention, which included the following 4 tools: Satisfaction and knowledge questionnaires (Appendices A + B), Pain relief Protocol (Appendix C) and Weekly Training sessions (Appendix D).

\section{Stage 2- Data gathering of KSA pre analysis}

Prior to the educational intervention, anonymous KSA questionnaires were sent to all the physicians participating in the study.

\section{Stage 3- Education implementation}


A course of ten sessions was held during 3 months which focused on different aspects of pain management. In addition, a new pain relief protocol was introduced in the first educational sessions and physicians began to prescribe treatment according to the protocol. Pain trusties supervised the entire intervention

\section{Stage 4- Intervention period +data gathering of KSA- post analysis}

Following the completion of the intervention period, the same questionnaires were given again to examine the objective and subjective improvement of the educational process.

\section{Research tools:}

KSA questionnaires: (Appendices A +B) The purpose was to examine the effectiveness of the educational intervention among the physicians participating in the study. The Likert-type scale ${ }^{9}$ was used to assess physician reflection on the process. Satisfaction Scale ranged from: $1=$ strongly Disagree, $2=$ Disagree, 3 $=$ Disagree to a certain extent, $4=$ Agree to a certain extent, $5=$ Agree, $6=$ strongly agree .

The physicians were asked to indicate the extent of their agreement with the range of statements regarding their satisfaction with the department's conduct and their personal conduct in dealing with a painful patient.

Part B was a Likert-type modified "KnowPain50" [KP50] questionnaire ${ }^{10}$. The KP50 helps to assess the level of knowledge and approach of physicians to pain management. By using this tool, it became possible to assess the gaps in the level of professional knowledge, the general approach to pain and how it should be treated.

KP50 modified knowledge questionnaire, included 15 selected questions that were found to be most relevant to internal medicine physician, from the original KP50 questionnaire.

\section{Intervention tools :}

The intervention tool was the educational intervention that included the following:

1. Pain Relief Protocol (Appendix C) -This Protocol was a pocket pamphlet distributed to all physicians in the department. It was based on the drug availability from the hospital's pharmacy. In the protocol, treatment was divided according to three levels of pain: mild, moderate and severe, each of which were categorized into acute and chronic treatment ("Chronic" referred to pain relief treatment administrated around the clock during the patient's stay in the department). In addition, the protocol included a section regarding neuropathic pain, an opiate conversion table and their possible side effects.

2. Weekly training sessions (Appendix D) - 10 sessions of 90 minutes each. These ssions included a general approach to the painful patient, familiarity with the common types of pain encountered by physicians within the internal department and the pharmacology of pain medications. Emphasis was placed on drug therapy in the internal medicine department for common conditions such as oncological, musculoskeletal, visceral pain etc.

\section{The study variables:}

The variables in the study are personal and departmental satisfaction regarding the management of the painful patient, and a knowledge questionnaire on various subjects related to pain management, within the framework of an interna lmedicine department.

\section{Statistical analysis:}

Statistical analysis was performed using SPSS Version 21. Since the study population was relatively small, the data was not distributed normally, thus an a-parametric test was chosen, "Wilcoxon Signed Ranks test" to examine the differences between the variables, related to satisfaction in pain management. Variables related to the pain knowledge parameter were examined by the Mc-Nemar test. P.Value of less than 0.05 was consideredstatistically significant.

Results 


\section{Part A- satisfaction questionnaire}

Overall satisfaction (table 1):

The overall satisfaction of the physicians from their pain management practice in the ward improved significantly following the educational intervention (2.83 to 4.4, p.value 0.003$)$.Moreover the majority of the physicians' satisfaction was inverted from a negative point of view to a positive one (median 2.7 to 4.5 ).

Departmental satisfaction (table 2):

The departmental satisfaction of its pain management significantly improved in more than 2 points in satisfaction level scale (3.01 to 5.09, p.value $=0.003)$. The biggest changes in satisfaction from department performance were in pain monitoring (question 5-median improved in 4 satisfaction levels) and the pain assessment (question 9- median improved in 2 satisfaction levels)

\section{Personal Satisfaction (Table 3):}

The physicians' personal satisfaction levels regarding their personal skill in managing a painful patient was significantly improved by 1.39 points (p.value $=0.003$ ) in the satisfaction level scale. The most notable improvement is that physicians took a personal responsibility for their patient's pain monitoring and performed daily pain assessments (questions 2+4: a rise in two satisfaction points in the satisfaction level scale). There was a notable rise in confidence in the approach of prescribing opiates following the educational process (question 10- p.value $=0.011$ ).

\section{Part B-A modified KP50 questionnaire}

Table 4 describes the results of a knowledge questionnaire on various subjects related to pain physiology, pain management, and side effects of drug therapy (see Appendix C). This part was based on selected questions from the KP50 questionnaire. A perfect score is 75. Prior to educational intervention, the average score for all physicians is $45.7 / 75$ with a median of $46 / 75$.

After the intervention, a significant improvement was observed as the mean score increased to $52.70 / 75$ with a median of $55 / 75$ (p.value $=0.012)$.

\section{Discussion:}

"There is more than enough pain around, and no pain should be added, it is worthwhile, if possible, to reduce it, not to sprinkle more salt on open wounds"; Writes author Amos Oz, in his book "A Perfect Peace" 13 . Our medical altruism always strives to help reduce the suffering and pain that exists among our patients. Unfortunately there is a big gap between the therapeutic ideal and the actual pain treatment. Consequently, the frustration is on both sides- physicians' and patients' alike. This gap, as observed in previous studies is mainly due to insufficient knowledge, and lack of efficient work protocols.

In this study, an educational intervention was mostly associated with an increase in the doctor's satisfaction from the department performance. The educational process encouraged new norms in the department with regard to pain monitoring, which is the first and essential stage in the treatment of the painful patient. The implementation of the educational intervention manifests by itself a commitment to the process; a department that chooses to secure time for this process recognizes the gaps that exist within it and essentially declares that it is committed to responding to the pain experienced by their patients. Our research shows that the commitment that a department takes upon itself yields fruit and permeates to the entire medical staff in the department.

The personal satisfaction of doctors increased significantly but not, as much as we expected. The reasons for this may be that the educational intervention is deficient, and certain content must be strengthened and practiced.

The level of knowledge regarding pain relief, increased and improved among the medical staff, thus providing a basic and important step in the will to close the gap and perhaps to improve pain treatment in the internal 
medicine department. It is important to note that the median score was higher than the mean, meaning that the improvement of knowledge was for most doctors, which reinforces the change that has emerged following the educational intervention.

The opioid crisis is one of the most urgent medical problems in the USA ${ }^{12}$. An improtant lesson from the opioid crisis is that educational intervention should be dynamic and address changes in the concept of drug therapy. A significant achievement of the educational intervention was the rise in confidence and knowledge among physicians in prescribing opiates. Such change in confidence and knowledge in prescribing opiates may bring to a better tailored pain relief treatment with less side effects. It is advised to continue with a clinical research to track whether the actual treatment of patients was more accurate and with fewer side effects after opioid treatment.

The prevalence and complications of diabetes and neuropathic pain have been on a rise in recent years ${ }^{13}$. Unfortunately, a lack of improvement in knowledge for neuropathic pain was observed in the knowledge questionaire, thus indicating a weak point in the educational process that needs to be improved in future educational interventions.

In order to maintain the standards of care following the educational program we recommend setting up a dedicated team of pain trustees that would maintain the quality of pain relief treatment and perform a routinely repetitive educational process as needed.

\section{Research Limitations}

This study examined an educational process at a specific period in time ("snapshot") that lasted a few months. The results shows a significant improvement in most of the parameters examined in the study. It is important to repeat the questionnaires a year after the intervention in order to examine the long-term impact of such process and to observe whether the norms we tried to instill in the department did indeed seep into the work of the medical staff in the department.

Two important points regarding our knowledge assessment tool are that, first we use only parts of the original KP50 questionnaire so it is without validation. Secondly, since the KP50 was developed and validated before the eruption of the opioid crisis, we think that there might be a place to revise the questionnaire to address the changes in opioids prescription following this pandemic.

The medical experience and academic background of the physicians participating in the study was not uniform. The physicians participating in the study were in different stages of their residency program, have studied in different faculties, some in Israel and some abroad. The different background can explain the change in the median. In order to eliminate this bias, the study examined individual change for each physician, but the main inference is that the education program needs to adapt to different backgrounds of doctors.

\section{In conclusion:}

Narrowing the gap in knowledge and dis-satisfaction can be performed by a short educational intervention. This simple intervention can be assimilated to other internal departments and also to other departments, after few adjustments. Future research, should focus on the practical outcomes in pain management.

\section{Bibliography:}

1. The Tragedy of Needless Pain. Scientific Americanhttps://www.scientificamerican.com/article/thetragedy-of-needless-pain/ doi:10.1038/scientificamerican0290-27.

2. Sinatra, R. Causes and consequences of inadequate management of acute pain. Pain Med. Malden Mass $11,1859-1871$ (2010).

3. Stalnikowicz, R., Mahamid, R., Kaspi, S. \& Brezis, M. Undertreatment of acute pain in the emergency department: a challenge. Int. J. Qual. Health Care J. Int. Soc. Qual. Health Care 17, 173-176 (2005). 
4. Baldemir, R. et al. [An assessment of physicians attitudes toward opioid usage and opiophobia: Results of a survey from a training and research hospital]. Agri Agri Algoloji Derneginin Yayin Organidir J. Turk. Soc. Algol. 31 , 23-31 (2019).

5. Gregory, J. \& McGowan, L. An examination of the prevalence of acute pain for hospitalised adult patients: a systematic review. J. Clin. Nurs. 25 , 583-598 (2016).

6. Ponte, C. D. \& Johnson-Tribino, J. Attitudes and knowledge about pain: an assessment of West Virginia family physicians. Fam. Med.37 , 477-480 (2005).

7. Weinstein, S. M. et al. Physicians' Attitudes Toward Pain and the Use of Opioid Analgesics: Results of a Survey from the Texas Cancer Pain Initiative. South. Med. J. 93 , 479-487 (2000).

8. Gaskin, D. J. \& Richard, P. The economic costs of pain in the United States. J. Pain Off. J. Am. Pain Soc. $13,715-724$ (2012).

9. Sullivan, G. M. \& Artino, A. R. Analyzing and interpreting data from likert-type scales. J. Grad. Med. Educ. 5 , 541-542 (2013).

10. Harris, J. M. et al. KnowPain-50: a tool for assessing physician pain management education. Pain Med. Malden Mass9 , 542-554 (2008).

11. A Perfect Peace. https://www.goodreads.com/work/best_book/523981.

12. Hodge, J. G. et al. Innovative Law and Policy Responses to the Opioid Crisis. J. Law. Med. Ethics $\mathbf{4 7}$ , 173-176 (2019).

13. Fayaz, A., Croft, P., Langford, R. M., Donaldson, L. J. \& Jones, G. T. Prevalence of chronic pain in the UK: a systematic review and meta-analysis of population studies. BMJ Open 6 , e010364 (2016).

Figure 1 - Flowchart of research methodology

Table 1 - Overall satisfaction

Table 2 - Departmental satisfaction

Table No. 3 - Personal Satisfaction

Table 4 - Results of the KP50 based knowledge questionnaire

\section{Hosted file}

Figures.docx available at https://authorea.com/users/421359/articles/527423-improvingpain-management-in-an-hospital-ward-by-implementing-an-educational-intervention-aprospective-cohort-study

\section{Hosted file}

Table 1.docx available at https://authorea.com/users/421359/articles/527423-improvingpain-management-in-an-hospital-ward-by-implementing-an-educational-intervention-aprospective-cohort-study

\section{Hosted file}

Table 2.docx available at https://authorea.com/users/421359/articles/527423-improvingpain-management-in-an-hospital-ward-by-implementing-an-educational-intervention-aprospective-cohort-study

\section{Hosted file}


Table 3.docx available at https://authorea.com/users/421359/articles/527423-improvingpain-management-in-an-hospital-ward-by-implementing-an-educational-intervention-aprospective-cohort-study

\section{Hosted file}

Table 4.docx available at https://authorea.com/users/421359/articles/527423-improvingpain-management-in-an-hospital-ward-by-implementing-an-educational-intervention-aprospective-cohort-study 